\title{
One- and two-neutron removal reactions from the most neutron-rich carbon isotopes
}

\author{
N. Kobayashii, ${ }^{1}$ T. Nakamura, ${ }^{1}$ J. A. Tostevin, ${ }^{1,2}$ Y. Kondo, ${ }^{1}$ N. Aoi,${ }^{3}$ H. Baba, ${ }^{3}$ S. Deguchi,,${ }^{1}$ J. Gibelin, ${ }^{4}$ M. Ishihara, ${ }^{3}$ \\ Y. Kawada, ${ }^{1}$ T. Kubo, ${ }^{3}$ T. Motobayashi, ${ }^{3}$ T. Ohnishi, ${ }^{3}$ N. A. Orr, ${ }^{4}$ H. Otsu, ${ }^{3}$ H. Sakurai, ${ }^{3}$ Y. Satou, ${ }^{5}$ E. C. Simpson, ${ }^{2}$ \\ T. Sumikama, ${ }^{6}$ H. Takeda, ${ }^{3}$ M. Takechi, ${ }^{3}$ S. Takeuchi, ${ }^{3}$ K. N. Tanaka, ${ }^{1}$ N. Tanaka, ${ }^{1}$ Y. Togano, ${ }^{3}$ and K. Yoneda ${ }^{3}$ \\ ${ }^{1}$ Department of Physics, Tokyo Institute of Technology, 2-12-1 O-Okayama, Meguro, Tokyo 152-8551, Japan \\ ${ }^{2}$ Department of Physics, Faculty of Engineering and Physical Sciences, University of Surrey, Guildford, Surrey GU2 7XH, United Kingdom \\ ${ }^{3}$ RIKEN Nishina Center, Hirosawa 2-1, Wako, Saitama 351-0198, Japan \\ ${ }^{4}$ LPC-Caen, ENSICAEN, IN2P3-CNRS, Université de Caen, 14050, Caen Cedex, France \\ ${ }^{5}$ Department of Physics and Astronomy, Seoul National University, 599 Gwanak, Seoul 151-742, Korea \\ ${ }^{6}$ Department of Physics, Tokyo University of Science, Chiba 278-8510, Japan
}

(Received 30 November 2011; published 5 November 2012)

\begin{abstract}
The structure of ${ }^{19,20,22} \mathrm{C}$ has been investigated using high-energy (around $240 \mathrm{MeV} /$ nucleon) one- and two-neutron removal reactions on a carbon target. Measurements were made of the inclusive cross sections and momentum distributions for the charged residues. Narrow momentum distributions were observed for one-neutron removal from ${ }^{19} \mathrm{C}$ and ${ }^{20} \mathrm{C}$ and two-neutron removal from ${ }^{22} \mathrm{C}$. Two-neutron removal from ${ }^{20} \mathrm{C}$ resulted in a relatively broad momentum distribution. The results are compared with eikonal-model calculations combined with shell-model structure information. The neutron removal cross sections and associated momentum distributions are calculated for transitions to both the particle-bound and particle-unbound final states. The calculations take into account the population of the mass $A-1$ reaction residues ${ }^{A-1} \mathrm{C}$ and, following one-neutron emission after one-neutron removal, the mass $A-2$ two-neutron removal residues ${ }^{A-2} \mathrm{C}$. The smaller contributions of direct two-neutron removal, that populate the ${ }^{A-2} \mathrm{C}$ residues in a single step, are also computed. The data and calculations are shown to be in good overall agreement and consistent with the predicted shell-model ground-state configurations and one-neutron overlaps with low-lying states in ${ }^{18-21} \mathrm{C}$. These suggest significant $\nu s_{1 / 2}^{2}$ valence neutron configurations in both ${ }^{20} \mathrm{C}$ and ${ }^{22} \mathrm{C}$. The results for ${ }^{22} \mathrm{C}$ strongly support the picture of ${ }^{22} \mathrm{C}$ as a two-neutron halo nucleus with a dominant $\nu s_{1 / 2}^{2}$ ground-state configuration.
\end{abstract}

DOI: 10.1103/PhysRevC.86.054604

PACS number(s): 24.50.+g, 21.10.Jx, 25.60.Gc, 27.20.+n

\section{INTRODUCTION}

Residue momentum distributions following the dissociation of halo nuclei have long been recognized as probes of the spatially extended valence neutron wave functions [1,2]. Over the last decade, high-energy nucleon removal reactions have become a powerful spectroscopic tool to explore the structure of nuclei far from stability. Specifically, the momentum distributions and associated cross sections offer a means to probe the active single-particle orbitals near the Fermi surface, whereby the shapes of the momentum distributions reflect the orbital angular momentum of the removed nucleon(s) and cross sections the spectroscopic strengths [3-8].

Two-nucleon $(2 N)$ removal reactions are challenging experimentally and remain less well studied [9-12]. Recently, it has been clarified that $2 N$ removal cross sections and associated residual nucleus momentum distributions, following direct removal of two well-bound like nucleons, can provide a sensitive probe of rapid structural changes [13,14], of spins of final states [15,16], and also of aspects of two-nucleon spatial correlations near the nuclear surface [12,17]. However, a complication arises when discussing reactions that result in the removal of two weakly bound (valence) neutrons from a neutron-rich mass $A$ projectile. The mass $A-2$ reaction residues can now result from two distinct mechanisms: singlestep direct knockout of a pair of neutrons and one-neutron knockout followed by neutron emission from excited particleunbound states of the intermediate mass $A-1$ residue. The latter will be populated with cross sections that are typically an order of magnitude or more larger than those for the direct pair removal. Both processes are discussed quantitatively in the analyses presented here.

This article reports measurements of one and twoneutron removal from ${ }^{19,20,22} \mathrm{C}$ secondary beams at around $240 \mathrm{MeV} /$ nucleon. The carbon isotopic chain is of considerable interest from a structural point of view, as it exhibits large odd-even staggering in the one-neutron separation energies (see, e.g., Fig. 2 of Ref. [18]), and weak $s$-wave valence neutron binding and halo formation for both ${ }^{15} \mathrm{C}$ [19], $S_{1 n}=$ 1.22 (1) $\mathrm{MeV}$, and ${ }^{19} \mathrm{C}$ [20], $S_{1 n}=0.58(9) \mathrm{MeV}$. The next heavier odd- $A$ halo candidate, ${ }^{21} \mathrm{C}$, is known to be particle unbound, while ${ }^{22} \mathrm{C}$ is bound by $S_{2 n}=0.42$ (94) $\mathrm{MeV}$, which is estimated on the basis of systematics [21]. As such, ${ }^{22} \mathrm{C}$ may be pictured as a three-body $\left({ }^{20} \mathrm{C}+n+n\right)$ Borromean system, having no bound two-body subsystems.

A recent measurement of the reaction cross section of ${ }^{22} \mathrm{C}$ on a proton target [22] has also drawn much attention. Its observation of an enhanced cross section suggests that ${ }^{22} \mathrm{C}$ has (a) an extended matter density and (b) a two-neutron halo ground-state structure, dominated by a weakly bound $s$-wave two-neutron configuration. Such measurements, carried out over a wide energy range, have been successful in deducing nuclear matter radii and their evolution along isotopic chains. However, the radii alone do not allow the detailed changes in the microscopic structure to be deduced, and additional, more exclusive, measurements are needed to do so. 
Fast one- and two-neutron removal reactions from the lighter neutron-rich carbon isotopes, up to ${ }^{19} \mathrm{C}$, have been studied experimentally using beryllium and carbon targets (see, e.g., [19,23-31]). In several cases, the parallel or transverse momentum distributions of the reaction residues were also measured. The beam energies of these studies ranged from 43-916 MeV/nucleon. A systematic analysis using the shell model and eikonal reaction formalism exploited here was presented in Ref. [18]. Results for neutron removal from ${ }^{18,19} \mathrm{C}$ on a proton target at $68 \mathrm{MeV} /$ nucleon were reported by Kondo et al. [32] whereby the transverse momentum distributions were interpreted within the context of continuumdiscretized coupled-channels (CDCC) calculations. Such an approach was required to treat the proton-induced reactions whereby the target may not be treated as a strongly absorptive ("black-disk"-like) object. More recently, Ozawa et al. [33] have investigated one- and two-neutron removal reactions of ${ }^{19,20} \mathrm{C}$ at $40 \mathrm{MeV} /$ nucleon using a proton target. The results for $\left({ }^{19} \mathrm{C},{ }^{18} \mathrm{C}\right)$ could be reproduced using a Glauber-type model analysis incorporating shell-model input, while the one- and two-neutron removal cross sections of ${ }^{20} \mathrm{C}$ could not.

The measurements reported here were made at around $240 \mathrm{MeV} /$ nucleon. At such an energy, the underlying assumptions of the sudden and eikonal approximations for the reactions are well founded. This paper thus presents a quantitative analysis of the results of both (a) direct one- and two-neutron removal, and (b) indirect two-neutron removal. In doing so, we are able to elucidate the dominant single-particle structure of ${ }^{19,20,22} \mathrm{C}$ and the extent to which these are tracked by shell-model calculations at the boundary of the $p$ shell and the lower part of the $s d$ shell. Only the shell model currently provides this level of information on the nucleon single-particle structures consistently along an isotopic chain in a form that can be readily used in reaction calculations. Thus, we employ a consistent shell-model and reaction theory description for all of the isotopes considered.

In Sec. II we discuss the experimental techniques. The results are described in Sec. III. The theoretical approach used is outlined in Sec. IV. There, both the reaction theory and shell-model spectroscopic strengths and description of the neutron bound states needed for the calculations are discussed. The experimental results and calculations are compared and discussed in Sec. V. The paper then concludes with a brief summary.

\section{EXPERIMENTAL SETUP AND DETAILS}

The experiments were performed at the RI Beam factory (RIBF) operated by the RIKEN Nishina Center and the Center for Nuclear Study, University of Tokyo. Secondary beams of ${ }_{19,20,22} \mathrm{C}$ were produced by fragmentation of a ${ }^{48} \mathrm{Ca}$ beam at $345 \mathrm{MeV} /$ nucleon on a 20-mm-thick rotating Be target. The typical ${ }^{48} \mathrm{Ca}$ primary beam intensities $\left(I_{1}\right)$ for each setting are listed in Table I. The secondary beams were separated using the superconducting separator BigRIPS [34,35], the layout of which is shown in Fig. 1. An achromatic aluminum energy degrader of 15-mm-median thickness was located at the dispersive focal plane F1. For the setting to produce the ${ }^{19} \mathrm{C}$
TABLE I. The typical ${ }^{48}$ Ca primary beam intensity $\left(I_{1}\right)$ for each setting and typical secondary beam intensity $\left(I_{2}\right)$. Also tabulated are the momentum spread $(\Delta P / P)$ of the secondary beams.

\begin{tabular}{lccc}
\hline \hline Secondary beam & $I_{1}(\mathrm{pnA})$ & $I_{2}($ particles $/ \mathrm{s})$ & $\Delta P / P$ \\
\hline${ }^{19} \mathrm{C}$ & $\approx 4$ & $\approx 1 \times 10^{3}$ & $\pm 2 \%$ \\
${ }^{20} \mathrm{C}$ & $\approx 6$ & $\approx 6 \times 10^{2}$ & $\pm 3 \%$ \\
${ }^{22} \mathrm{C}$ & $\approx 80$ & $\approx 6$ & $\pm 3 \%$ \\
\hline \hline
\end{tabular}

beam, an aluminum degrader of 8-mm-median thickness was also installed at the dispersive focal plane F5. The secondary beam particles were identified event by event by combining the measured time of flight (TOF), energy loss $(\Delta E)$, and magnetic rigidity $(B \rho)$. The TOF was recorded between two plastic scintillators at the achromatic focal planes F3 and F7, $\Delta E$ was measured using a plastic scintillator at $\mathrm{F} 7$, and $B \rho$ was determined from a position measurement using a plastic scintillator readout on both sides by photomultiplier tubes at F5. An example of the particle-identification spectrum is shown in Fig. 2(a) in terms of the atomic number $(Z)$ and mass-to-charge ratio $(A / Z)$, which demonstrates the clear separation of ${ }^{22} \mathrm{C}$. The secondary beam intensities $\left(I_{2}\right)$ and momentum spread $(\Delta P / P)$ of the ${ }^{19,20,22} \mathrm{C}$ beams are listed in Table I. A secondary carbon target $\left(4.02 \mathrm{~g} \mathrm{~cm}^{2}\right)$ was mounted at the achromatic focal plane F8. The mid-target energies $(\bar{E} / A)$ of ${ }^{19,20,22} \mathrm{C}$ were 243,241 , and $240 \mathrm{MeV} /$ nucleon, respectively, as listed in Table II.

The ${ }^{18,19,20} \mathrm{C}$ residues following the reaction were collected by tuning the rigidity of the zero- degree spectrometer (ZDS) to center the momentum distribution. The residues as well as the secondary beam particles were identified event by event by combining TOF, $\Delta E$, and $B \rho$. The TOF of the reaction products (F8-F11) was determined by subtracting from the F7-F11 TOF the TOF for the beam particles between F7 and F8. The latter was based on the velocity determined from the TOF measured between F3 and F7. We note that no timing scintillator was installed near the F8 target position in order to reduce any backgrounds arising from reactions on materials other than the target. The energy loss $\Delta E$ was measured using an ionization chamber at F11. The magnetic rigidity $B \rho$ was determined from a position measurement using parallel plate avalanche

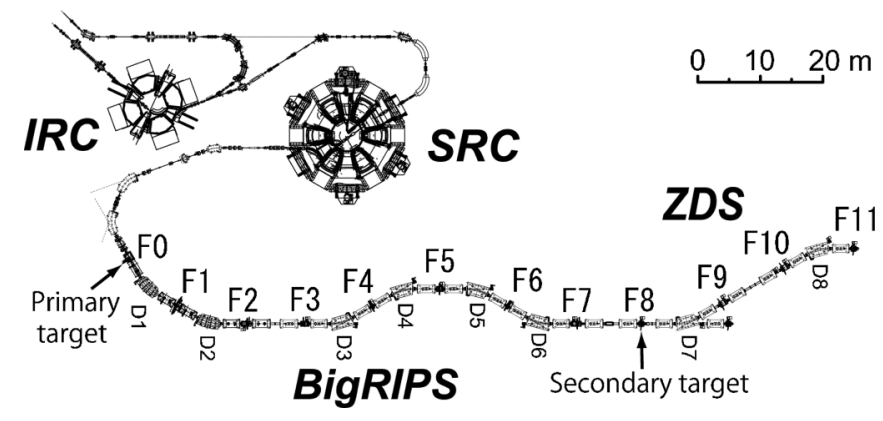

FIG. 1. A schematic view of the layout of BigRIPS (F0-F8) and zero-degree spectrometer (ZDS) (F8-F11), where F1 through F11 represent each focal plane. The secondary carbon target was mounted at the achromatic focal plane $\mathrm{F} 8$. The ${ }^{18,19,20} \mathrm{C}$ residues were identified using the ZDS. 


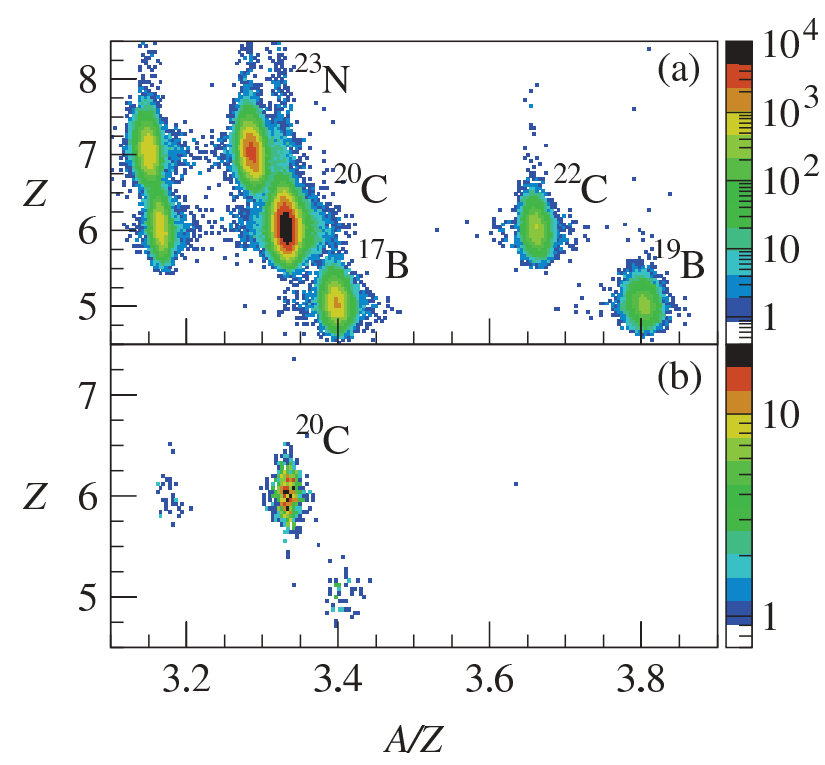

FIG. 2. (Color online) Particle-identification spectrum of (a) the ${ }^{22} \mathrm{C}$ beam by BigRIPS, and (b) the residues in the ZDS after selecting ${ }^{22} \mathrm{C}$ ions before the secondary target.

counters (PPAC) at the dispersive focal plane F9. The resulting particle-identification spectrum for the ${ }^{20} \mathrm{C}$ residues is shown in Fig. 2(b).

This study measured one- and two-neutron removal cross sections $\left(\sigma_{-x n}^{\exp }, x=1\right.$ or 2$)$ and residue parallel momentum $\left(P_{||}\right)$distributions for the channels listed in Table II. The cross sections were derived from the numbers of the beam particles counted before the secondary target and those of the residues registered at the final focal plane F11 of the ZDS, using

$$
\sigma_{-x n}^{\exp }=\left(\frac{N_{i}^{\prime}}{N_{i}}-\frac{N_{o}^{\prime}}{N_{o}}\right)\left(\frac{\sigma_{R}-\sigma_{R}^{\prime}}{e^{-\sigma_{R}^{\prime} N_{t}}-e^{-\sigma_{R} N_{t}}}\right) .
$$

Here, $N_{i}\left(N_{o}\right)$ represents the number of projectiles and $N_{i}^{\prime}$ $\left(N_{o}^{\prime}\right)$ the number of residues for target-in $(i)$ [target-out $(o)$ ] runs. $N_{t}$ and $\sigma_{R}\left(\sigma_{R}^{\prime}\right)$ are the number of target nuclei per unit area and reaction cross section of the projectile (residue). The background events were subtracted by using target-out runs. Owing to the substantial thickness of the carbon target, such a correction had to be applied to account for the losses in the number of projectiles and residues owing to reactions in the target. To do so, total reaction cross sections of $1375 \mathrm{mb}$ for ${ }^{22} \mathrm{C}, 1090 \mathrm{mb}$ for ${ }^{20} \mathrm{C}, 1139 \mathrm{mb}$ for ${ }^{19} \mathrm{C}$, and $1023 \mathrm{mb}$ for ${ }^{18} \mathrm{C}$, which were estimated using eikonal calculations, were

TABLE II. The one- and two-neutron removal cross sections for each reaction channel at the mid-target energies $(\bar{E} / A)$ are shown.

\begin{tabular}{lcr}
\hline \hline Reaction & $\bar{E} / A(\mathrm{MeV})$ & $\sigma_{-x n}^{\exp }(\mathrm{mb})$ \\
\hline $\mathrm{C}\left({ }^{19} \mathrm{C},{ }^{18} \mathrm{C}\right)$ & 243 & $163(12)$ \\
$\mathrm{C}\left({ }^{20} \mathrm{C},{ }^{19} \mathrm{C}\right)$ & 241 & $58(5)$ \\
$\mathrm{C}\left({ }^{20} \mathrm{C},{ }^{18} \mathrm{C}\right)$ & 241 & $155(25)$ \\
$\mathrm{C}\left({ }^{22} \mathrm{C},{ }^{20} \mathrm{C}\right)$ & 240 & $266(19)$ \\
\hline \hline
\end{tabular}

employed. It should be noted that a $10 \%$ deviation in these reaction-cross-section values translates into an essentially negligible $1 \%$ deviation of the deduced one- and two-neutron removal cross sections. To obtain the residue momentum in the projectile rest frame, the Lorentz transformation was applied to the laboratory system residue momentum derived from the TOF between F8 and F11, whereby corrections have been applied to account for the different flight paths. Similarly, the velocity of the projectile was deduced from the TOF between F3 and F7. The cross sections $\sigma_{-x n}^{\exp }$ and parallel momentum $P_{\|}$distributions were corrected for the transmission efficiency through the ZDS. These were estimated using a Monte Carlo simulation, an angular acceptance of the ZDS obtained by calibration runs using the secondary beams, and design value of the momentum acceptance $(\Delta P / P \leqslant \pm 3 \%)$ of the ZDS. To obtain a higher transmission, the momentum acceptance of BigRIPS was restricted to $\Delta P / P \leqslant \pm 2 \%$ in the analysis. The transmission was over $90 \%$ in all cases.

\section{EXPERIMENTAL RESULTS}

The one- and two-neutron removal cross sections extracted here are summarized in Table II together with the corresponding mid-target energy of the projectile. The cross section for $\mathrm{C}\left({ }^{19} \mathrm{C},{ }^{18} \mathrm{C}\right)$ is smaller than that measured at lower energies. For instance, at $64 \mathrm{MeV} /$ nucleon, the cross section $\sigma_{-1 n}$ is $226(65) \mathrm{mb}$ [30], about $40 \%$ larger than the current value. In part, this reflects the smaller intrinsic nucleon-nucleon cross sections at the energy of the current experiment (being closer to the minimum near $300 \mathrm{MeV}$ ) as well as changes in the real parts of the optical potentials and a reduced diffractive breakup contribution for energies in excess of $100 \mathrm{MeV} /$ nucleon [36]. For ${ }^{20} \mathrm{C}$, the yield of ${ }^{19} \mathrm{C}$ residues is much smaller, by a factor of about 3 , than that of ${ }^{18} \mathrm{C}$. We note that the recent measurement of one- and two-neutron removal from ${ }^{20} \mathrm{C}$ on a proton target at $40 \mathrm{MeV} /$ nucleon [33] showed a similar trend, where the cross section for one-neutron removal [22(8) $\mathrm{mb}$ ] is about $1 / 5$ of that for two-neutron removal [107(15) mb]. As will be shown later (Sec. V A2), the final states play a significant role in the cross sections. For ${ }^{22} \mathrm{C}$, we note an enhanced cross section compared to ${ }^{20} \mathrm{C}$ which, as we will show, reflects the two-neutron halo character of ${ }^{22} \mathrm{C}$.

The measured momentum distributions in the rest frame of the projectile are shown in Figs. 3-6. We note that $\left({ }^{22} \mathrm{C}\right.$, ${ }^{20} \mathrm{C}$ ) was obtained here. The broadening arising from the target effects and detector resolution was found to follow the Gaussian distribution with the $\sigma$ of 23,23, 28, and $27 \mathrm{MeV} / \mathrm{c}$ for $\left({ }^{19} \mathrm{C},{ }^{18} \mathrm{C}\right),\left({ }^{20} \mathrm{C},{ }^{19} \mathrm{C}\right),\left({ }^{20} \mathrm{C},{ }^{18} \mathrm{C}\right)$, and $\left({ }^{22} \mathrm{C}\right.$, ${ }^{20} \mathrm{C}$ ), respectively. The data show good agreement with the eikonal-model calculations (the details of which are described in Sec. IV) after folding with the experimental effects. Based on this comparison, the measured widths for $\left({ }^{19} \mathrm{C},{ }^{18} \mathrm{C}\right),\left({ }^{20} \mathrm{C}\right.$, $\left.{ }^{19} \mathrm{C}\right),\left({ }^{20} \mathrm{C},{ }^{18} \mathrm{C}\right)$, and $\left({ }^{22} \mathrm{C},{ }^{20} \mathrm{C}\right)$ are shown to be consistent with the FWHMs of $56,77,211$, and $73 \mathrm{MeV} / c$, respectively, obtained from the calculated distributions. We note that in order to derive any structural information, comparison should be made with the results of such realistic reaction theory as discussed in Sec. V. 


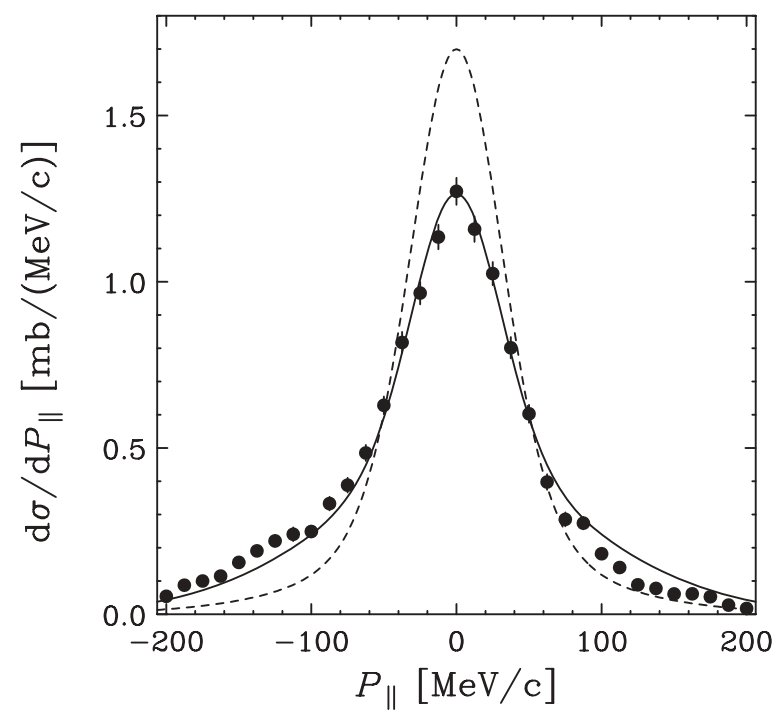

FIG. 3. Measured inclusive parallel momentum distribution of ${ }^{18} \mathrm{C}$, following one-neutron removal from ${ }^{19} \mathrm{C}$ on a carbon target at $243 \mathrm{MeV} /$ nucleon compared to the theoretical calculations. The solid line includes contributions from the $2_{3}^{+}$and $3_{1}^{+}$shell-model states of ${ }^{18} \mathrm{C}$, assumed bound (see also Table III). The dashed line shows the results when assuming that the $2_{3}^{+}$and $3_{1}^{+}$states are unbound. Here, and in Figs. 4-7, the theoretical distributions have been convoluted with the experimental resolution and normalized to the measured inclusive cross section.

It is clear that the momentum distributions for $\left({ }^{19} \mathrm{C},{ }^{18} \mathrm{C}\right)$, $\left({ }^{20} \mathrm{C},{ }^{19} \mathrm{C}\right)$, and $\left({ }^{22} \mathrm{C},{ }^{20} \mathrm{C}\right)$ are much narrower, at least by a factor of 2 , than those expected on the basis of the Goldhaber model $(210 \mathrm{MeV} / c$ for single-neutron removal, $290 \mathrm{MeV} / c$ for twoneutron removal) [37]. The narrow widths for the reactions on ${ }^{19} \mathrm{C}$ and ${ }^{22} \mathrm{C}$ arise from the weakly bound one- and two-neutron halolike nature of these nuclei and the role of $s$-wave valence neutron(s) in their ground-state structure. It is interesting that the measured distribution for $\left({ }^{20} \mathrm{C},{ }^{19} \mathrm{C}\right)$ is also narrow. This suggests that the reaction probes the $v s_{1 / 2}^{2}$ component of the ${ }^{20} \mathrm{C}\left(0^{+}\right)$ground-state wave function, as is required to populate the bound ${ }^{19} \mathrm{C}_{\text {g.s. }}\left(1 / 2^{+}\right)$halo state.

The momentum distribution for $\left({ }^{19} \mathrm{C},{ }^{18} \mathrm{C}\right)$ is consistent with a recent measurement [33] on protons at $40 \mathrm{MeV} /$ nucleon $[\mathrm{FWHM}=83(12) \mathrm{MeV} / \mathrm{c}]$, while that for $\left({ }^{20} \mathrm{C},{ }^{19} \mathrm{C}\right)$ is much narrower than the measurement [33] [FWHM = $168(20) \mathrm{MeV} / c]$. The cause of this difference is not entirely clear. We note, however, that the two measurements were made at very different energies on carbon and proton targets and, as such, are governed by different reaction mechanisms, being dominated by inelastic and elastic breakup mechanisms, respectively. Furthermore, the extraction of momentum distributions from measurements employing a zero-degree telescope [33] rather than a spectrometer to separate the reaction products (as was done here), requires subtraction of a strong component [dominant in the case of $\left({ }^{20} \mathrm{C},{ }^{19} \mathrm{C}\right)$ ] from reactions arising in the telescope itself. The quantitative analyses and interpretations of the momentum distributions and cross sections obtained here are detailed in the following sections.

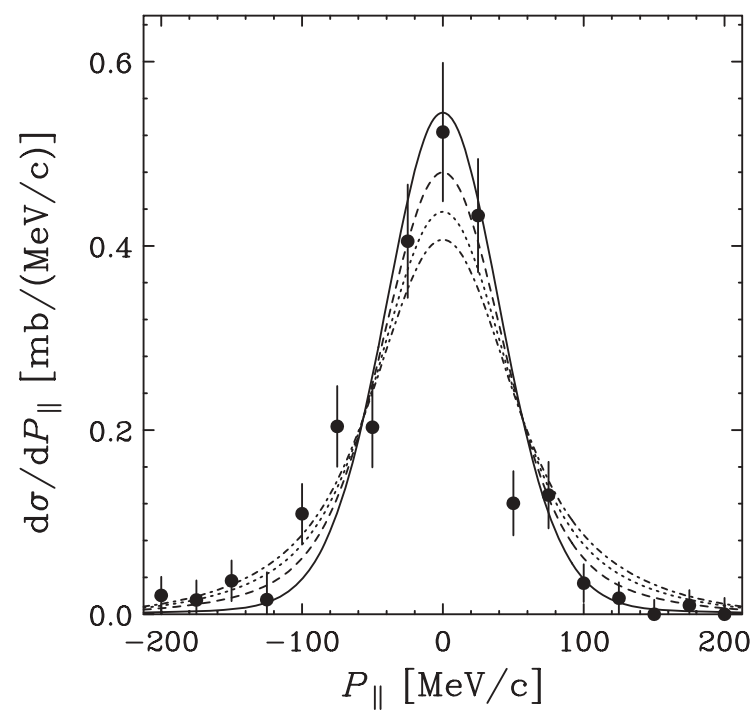

FIG. 4. Measured inclusive parallel momentum distribution of ${ }^{19} \mathrm{C}$, following one-neutron removal from ${ }^{20} \mathrm{C}$ on a carbon target at $241 \mathrm{MeV} /$ nucleon compared to the theoretical calculations. The solid curve assumes that only the $1 / 2^{+}$shell-model ground-state transition ( $2 s_{1 / 2}$ neutron removal) is bound. The long-dashed, short-dashed, and dotted-dashed curves result if one assumes that $0.5,1.0$, or 1.5 units of $1 d_{5 / 2}$ spectroscopic strength also lead to bound final states.

\section{REACTION ANALYSIS}

\section{A. Reaction theory}

We adopt an eikonal-model description of the reaction mechanisms. Given a nucleon- or nucleus-target interaction description, the eikonal approximation has been shown [38] to provide a rather accurate description of the elastic $S$ matrix and derived observables for incident projectile energies of order $50 \mathrm{MeV} /$ nucleon and greater. As noted earlier, at the energy of the current experiments (around $240 \mathrm{MeV} /$ nucleon), the sudden and eikonal approximations of the reaction model are very accurate.

The removal reaction cross sections for one-neutron knockout to a given final state, with spin-parity $J^{\pi}$, are calculated using [6]

$$
\sigma_{-1 n}=\sum_{n \ell j}\left[\frac{A}{A-1}\right]^{N} C^{2} S\left(J^{\pi}, n \ell j\right) \sigma_{\mathrm{sp}}\left(n \ell j, S_{n}^{\mathrm{eff}}\right),
$$

where the $C^{2} S$ are the shell-model spectroscopic factors and the single-particle cross section $\sigma_{\mathrm{sp}}$ is calculated using the eikonal model assuming unit spectroscopic factor. The quantum numbers of the removed neutron are denoted by $n \ell j$ and $S_{n}^{\text {eff }}$ is the effective separation energy for the removal of the neutron leaving the residue in the given final state. The singleparticle cross sections $\sigma_{\mathrm{sp}}$ include the contributions from both the stripping (inelastic breakup) and diffractive dissociation (elastic breakup) mechanisms. Details of calculations of these two distinct and (incoherent) additive contributions can be found in Ref. [39].

In direct two-neutron removal, the theoretical cross sections do not in general (e.g., when there are several active orbitals) 


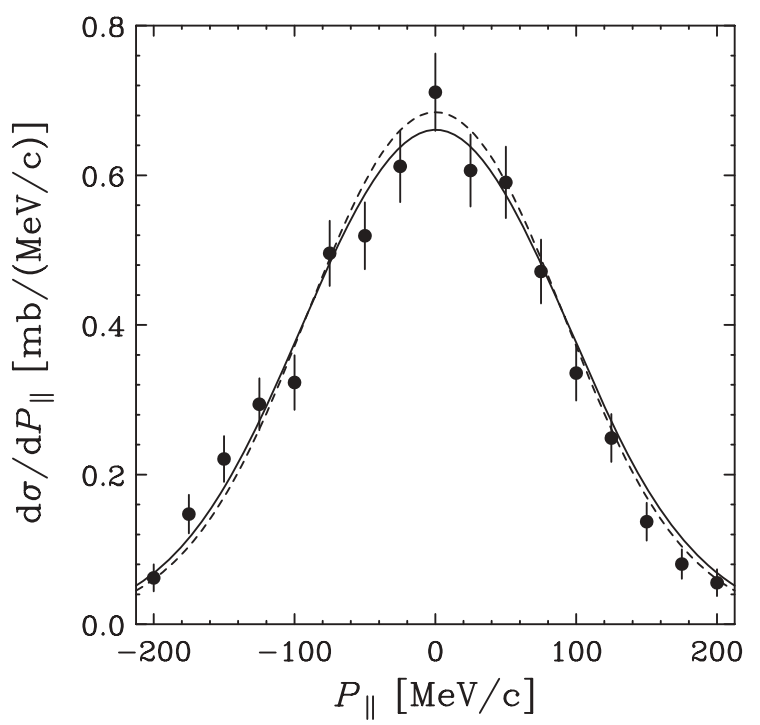

FIG. 5. Measured inclusive parallel momentum distribution of ${ }^{18} \mathrm{C}$, following two-neutron removal from ${ }^{20} \mathrm{C}$ on a carbon target at $241 \mathrm{MeV} /$ nucleon compared to the theoretical calculations. The theoretical curves are the weighted sum of the exclusive calculations of the unbound ${ }^{19} \mathrm{C}$ states (see text). Recoil effects associated with the neutron emission are included, assuming the most important contributions come from states with $\varepsilon^{*}$ of $1.0 \mathrm{MeV}$ (dashed curve) and 2.0 MeV (solid curve).

factorize into a structural (spectroscopic) factor and a singleparticle cross section. The cross sections involve coherent contributions from all active shell-model two-nucleon configurations with nonvanishing two-nucleon amplitudes (TNA). Details of their definitions and the phase conventions used can be found in Ref. [10]. Here, we will calculate the singlestep direct two-neutron removal yields arising from both (a) two-neutron stripping and (b) one neutron being stripped and the second being elastically dissociated (diffracted) [12]. Since these direct two-neutron removal cross sections are small compared to the cross sections arising from singleneutron removal, we do not expand upon these formal aspects here. Full details of the necessary eikonal formalism, as is applied to direct two-nucleon removal events, can be found in Refs. [10,12].

For both the one- and two-neutron removal calculations, the required neutron- and residue-target elastic $S$ matrices were calculated using the static density limit of the eikonal model, e.g., [40], also known as the t $\rho \rho$ limit of the Glauber multiple scattering series. That is, we used the single-folding model (nucleon target) and double-folding model (residue target) for the absorptive optical model interactions with the carbon target. The inputs needed were the residue and target point neutron and proton one-body densities and an effective nucleon-nucleon $(N N)$ interaction. The densities of the ${ }^{18,19,20,21} \mathrm{C}$ residues were estimated from spherical Skyrme Hartree-Fock (HF) calculations using the SkX interaction [41]. The HF calculates the (experimentally unbound) ${ }^{21} \mathrm{C}$ case to be weakly bound. We used this bound density for calculations of the ${ }^{21}$ C-target optical potential (and its $S$ matrix) in the localized region where they make grazing collisions.

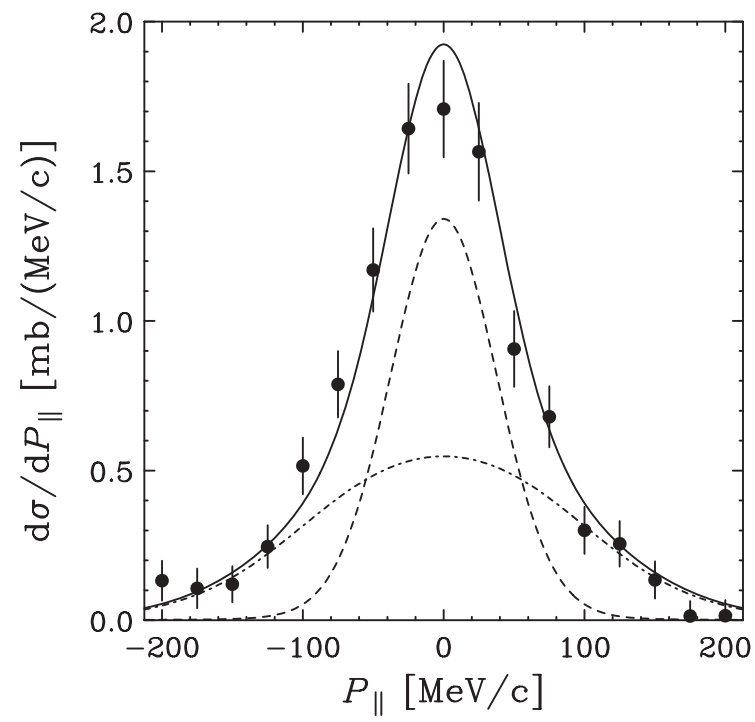

FIG. 6. Measured inclusive parallel momentum distribution of ${ }^{20} \mathrm{C}$, following two-neutron removal from ${ }^{22} \mathrm{C}$ on a carbon target at $240 \mathrm{MeV} /$ nucleon compared to the theoretical calculations. The solid curve is the weighted sum of the exclusive calculations for the unbound ${ }^{21} \mathrm{C}$ states (see text). The dashed and dotted-dashed curves show the contributions from knockout via the $1 / 2_{1}^{+}$and $5 / 2_{1}^{+}$unbound ${ }^{21} \mathrm{C}$ intermediate states, respectively. The recoil broadening arising from neutron emission from these unbound intermediate states is folded in.

All calculations assumed the following. The density of the carbon target nuclei was taken to be of Gaussian form with a point-nucleon root-mean-squared radius of $2.32 \mathrm{fm}$. A zero-range $N N$ effective interaction was used with its strength calculated from the free neutron-neutron and neutron-proton cross sections at the beam energy and from the real-toimaginary ratios of the $N N$ forward scattering amplitudes at $240 \mathrm{MeV}$, interpolated (using a polynomial fit) from the values tabulated by Ray [42]. The use of these inputs, as a standard parameter set in the eikonal reaction model, was shown to accurately reproduce the recently measured [43] ratios of the diffraction to stripping reaction mechanism yields in the cases of ${ }^{8} \mathrm{~B}$ and ${ }^{9} \mathrm{C}$ induced proton removal reactions. A recent careful analysis by Bertulani and De Conti [44] confirms that corrections to this adopted procedure, due to Pauli blocking corrections to the $N N$ effective interaction, are negligible at the energy of this study.

We assume here that the heavy residue-target interactions and their $S$ matrices are diagonal with respect to the different final states of the residue, and thus that there is no reactioninduced dynamical excitation of the residues during the collision. For the odd- $A$ carbon projectiles, where different neutron orbitals $(n \ell j)$ may then contribute to a given $J^{\pi}$ final state, this implies that these different $n \ell j$ contributions are incoherent and should be summed.

\section{B. Shell-model calculations and overlaps}

A consistent set of shell-model calculations were used for the required level energies, spectroscopic factors, and 
TABLE III. Results for one-neutron removal reactions from ${ }^{19,20} \mathrm{C}$. Tabulated are the one-neutron removal cross sections to assumed bound shell-model states near and below the neutron thresholds in the mass $A-1$ systems ${ }^{18,19} \mathrm{C}$ of 4.18 and $0.58 \mathrm{MeV}$, respectively (see also the footnotes). The final theoretical cross sections $\sigma_{-1 n}^{\text {th }}$ include the center-of-mass correction factor $[A /(A-1)]^{N}$. The errors shown for the ratio of cross sections $R_{s}=\sigma_{-1 n}^{\exp } / \sigma_{-1 n}^{\text {th }}$ reflect only the errors quoted on the measurements.

\begin{tabular}{|c|c|c|c|c|c|c|c|c|}
\hline Reaction & $E_{\mathrm{x}}(\mathrm{MeV})$ & $J^{\pi}$ & $\ell$ & $\sigma_{\mathrm{sp}}(\mathrm{mb})$ & $C^{2} S$ & $\sigma_{-1 n}^{\text {th }}(\mathrm{mb})$ & $\sigma_{-1 n}^{\exp }(\mathrm{mb})$ & $R_{s}$ \\
\hline$\left[{ }^{19} \mathrm{C}\left(1 / 2^{+}\right),{ }^{18} \mathrm{C}\left(J^{\pi}\right)\right]$ & 0.000 & $0_{1}^{+}$ & 0 & 104.7 & 0.580 & 67.63 & & \\
\hline \multirow[t]{4}{*}{$S_{1 n}\left({ }^{19} \mathrm{C}\right)=0.58 \mathrm{MeV}$} & 2.114 & $2_{1}^{+}$ & 2 & 29.91 & 0.479 & 15.96 & & \\
\hline & 3.988 & $0_{2}^{+}$ & 0 & 39.35 & 0.319 & 13.97 & & \\
\hline & $4.915^{\mathrm{a}}$ & $3_{1}^{+}$ & 2 & 23.60 & 1.523 & 40.04 & & \\
\hline & $4.975^{\mathrm{a}}$ & $2_{3}^{+}$ & 2 & 23.50 & 0.922 & 24.15 & & \\
\hline$\left[{ }^{20} \mathrm{C}\left(0^{+}\right),{ }^{19} \mathrm{C}\left(J^{\pi}\right)\right]$ & $0.000^{\mathrm{b}}$ & $1 / 2_{1}^{+}$ & 0 & 48.37 & 1.099 & 58.92 & & \\
\hline$S_{1 n}\left({ }^{20} \mathrm{C}\right)=2.90 \mathrm{MeV}$ & & Inclusive & & & & 58.92 & $58(5)$ & $0.98(8)$ \\
\hline
\end{tabular}

${ }^{\text {aThe }}{ }^{18} \mathrm{C} 23$ and $3_{1}^{+}$states at 4.915 and $4.975 \mathrm{MeV}$ are assumed to be bound (see Sec. V A1).

${ }^{\mathrm{b}}$ There is no evidence from this work that the $E_{\mathrm{x}}=0.190 \mathrm{MeV}, 5 / 2_{1}^{+}$shell-model state in ${ }^{19} \mathrm{C}$ is bound. This state is included in Table IV and is treated as unbound.

two-nucleon amplitudes. These were performed using the code OXBASH [45]. The calculations used the Warburton and Brown (WBP) effective interaction [46] in a $p s d$-model space truncated to allow $0 \hbar \omega$ and $1 \hbar \omega$ excitations. The small center-of-mass correction factor, $[A /(A-1)]^{N}$, shown in Eq. (2), where $N$ is the principal oscillator model quantum number of the orbit of the removed nucleon [47], was applied to the (fixed-center) shell-model spectroscopic factors of all of the single-neutron removal calculations.

The low-lying shell-model levels and spectroscopic factors for the reaction products, ${ }^{18,19,20,21} \mathrm{C}$, are collected in Tables III and IV. The wave function of each of the removed-neutron bound states was calculated in a Woods-Saxon potential well of a fixed geometry. Following Ref. [18], a reduced radius parameter of $r_{0}=1.25 \mathrm{fm}$ and a diffuseness of $a_{0}=0.7 \mathrm{fm}$ were adopted.
The depths of the potential wells in each instance were adjusted to reproduce the neutron separation energy, taking into account the excitation energy of the final state. The ground-state-to-ground-state one- and two-neutron separation energies were taken from the 2003 mass evaluation [21]. The shell-model excitation energies of Tables III and IV were then used (without any adjustment) for all non-ground-state transition calculations. For the direct two-neutron removal calculations, each neutron was assumed to have a separation energy of half that for the two neutrons to the final state of interest.

\section{ANALYSIS AND DISCUSSION}

We discuss the results for the inclusive cross sections for one- and two-neutron removal from the ${ }^{19,20,22} \mathrm{C}$ isotopes. We consider in detail the calculated contributions from both

TABLE IV. Results for the indirect two-neutron removal reaction cross sections. Tabulated are the one-neutron removal cross sections to all predicted unbound $A-1$-body shell-model states with energies below the neutron threshold of the mass $A-2$ systems. That is, the neutron-unbound final states of the intermediate mass $A-1$ systems ${ }^{19,21} \mathrm{C}$, below 4.18 and $2.90 \mathrm{MeV}$, respectively. The final theoretical cross sections $\sigma_{-1 n(e)}^{\text {th }}$ include the center-of-mass correction factor $[A /(A-1)]^{N}$. The errors shown on the ratio of cross sections $R_{s}=\sigma_{-2 n}^{\text {exp }} / \sigma_{-1 n(e)}^{\text {th }}$ reflect only the errors quoted on the measurements. The $\sigma_{-1 n(e)}^{\text {th }}$ values do not include direct two-neutron breakup events, and the $R_{s}$ values represent upper limits.

\begin{tabular}{|c|c|c|c|c|c|c|c|c|}
\hline Reaction & $E_{\mathrm{x}}(\mathrm{MeV})$ & $J^{\pi}$ & $\ell$ & $\sigma_{\mathrm{sp}}(\mathrm{mb})$ & $C^{2} S$ & $\sigma_{-1 n(e)}^{\text {th }}(\mathrm{mb})$ & $\sigma_{-2 n}^{\exp }(\mathrm{mb})$ & $R_{s}$ \\
\hline \multirow[t]{5}{*}{$S_{1 n}\left({ }^{20} \mathrm{C}\right)=2.90 \mathrm{MeV}$} & 0.624 & $3 / 2_{1}^{+}$ & 2 & 26.34 & 0.247 & 7.20 & & \\
\hline & 1.541 & $5 / 2_{2}^{+}$ & 2 & 24.31 & 0.282 & 7.59 & & \\
\hline & 2.417 & $3 / 2_{1}^{-}$ & 1 & 22.27 & 0.689 & 17.00 & & \\
\hline & 3.284 & $3 / 2_{2}^{+}$ & 2 & 21.50 & 0.191 & 4.56 & & \\
\hline & & Inclusive & & & & 191.2 & $155(25)$ & $<0.81(13)$ \\
\hline$\left[{ }^{22} \mathrm{C}\left(0^{+}\right),{ }^{21} \mathrm{C}\left(J^{\pi}\right)\right]$ & 0.000 & $1 / 2_{1}^{+}$ & 0 & 89.35 & 1.403 & 137.55 & & \\
\hline \multirow[t]{3}{*}{$S_{1 n}\left({ }^{22} \mathrm{C}\right)=0.70 \mathrm{MeV}$} & 1.109 & $5 / 2_{1}^{+}$ & 2 & 29.39 & 4.212 & 135.87 & & \\
\hline & 2.191 & $3 / 2_{1}^{+}$ & 2 & 25.44 & 0.342 & 9.55 & & \\
\hline & & Inclusive & & & & 283.0 & $266(19)$ & $<0.94(7)$ \\
\hline
\end{tabular}

a There is no evidence from this work that the $5 / 2_{1}^{+}$shell-model state in ${ }^{19} \mathrm{C}$ is bound. It is assumed to be unbound. 
indirect and direct two-nucleon removal. The measured and calculated inclusive parallel momentum distributions are also discussed. In all cases, these are shown in the projectile rest frame. In the comparisons with the data, the theoretical momentum distributions, calculated using the stripping mechanism, have been convoluted with the Gaussian experimental resolution given in Sec. III and then scaled to the measured inclusive cross sections. Further discussion of the calculations of the parallel momentum distributions in the cases of the transitions to unbound final states is included when discussing these, i.e., for the ${ }^{20,22} \mathrm{C}$ cases.

\section{A. One-neutron removal reactions}

We first discuss the individual and the inclusive one-neutron removal cross sections to bound $\sigma_{-1 n}^{\text {th }}$ and unbound (neutron emitting) $\sigma_{-1 n(e)}^{\text {th }}$ states of the mass $A-1$ isotopes. The experimental and theoretical results of this work are collected in Tables III and IV. Also tabulated are the details of the relevant shell-model states, their energies, spins, parities, and spectroscopic factors $C^{2} S$. The overall ratios of the measured to the calculated inclusive one-neutron removal cross sections $R_{s}=\sigma_{-1 n}^{\exp } / \sigma_{-1 n}^{\text {th }}$ are also shown in the tables for each bound and unbound final-states data set.

\section{Results for ${ }^{19} \mathrm{C}$}

The case of ${ }^{19} \mathrm{C}$ provides a valuable link to the earlier work at lower energies, summarized in Ref. [18], and the related and more exclusive results using neutron knockout from a proton target [32]. From this work, calculated exclusive and experimental inclusive one-neutron removal yields from the ${ }^{19} \mathrm{C}\left(1 / 2^{+}\right)$ground state, with ground-state separation energy $S_{1 n}\left({ }^{19} \mathrm{C}\right)=0.58 \mathrm{MeV}$, are shown in Table III. The theoretical cross sections are shown for the six predicted positive parity ${ }^{18} \mathrm{C}$ final states.

In the case of $\left({ }^{19} \mathrm{C},{ }^{18} \mathrm{C}\right)$, the WBP shell-model calculation places several final states near to or between the one- and twoneutron threshold energies of 4.18 and $4.91 \mathrm{MeV}$, respectively, for ${ }^{18} \mathrm{C}$. Specifically, the third $2_{3}^{+}$and first $3_{1}^{+}$states at 4.915 and $4.975 \mathrm{MeV}$ have significant spectroscopic strengths and associated cross sections. Experimentally, recent work of Kondo et al. [32], on neutron knockout from ${ }^{19} \mathrm{C}$ on a proton target, observed gamma rays from a $\left(2^{+}, 3^{+}\right)$excited state (or states) near $4.0 \mathrm{MeV}$, the associated ${ }^{18} \mathrm{C}$ transverse momentum distribution being characteristic of an $\ell=2$ neutron removal. The earlier $\left({ }^{19} \mathrm{C},{ }^{18} \mathrm{C}\right)$ inclusive data analyses of Maddalena et al. [26] and Simpson and Tostevin [18] also assumed these $2_{3}^{+}$and $3_{1}^{+}$states near $4.9 \mathrm{MeV}$ were neutron bound, citing the results of shell-model calculations using a modified version of the WBT interaction [48].

We have also calculated the inclusive parallel momentum distributions, to bound final states, as the sum of the distributions to these individual final states weighted by the $\sigma_{-1 n}^{\text {th }}$ shown in Table III. As in earlier studies [18,26], the lowest $1^{-}$state (which the present shell-model calculations place at $4.942 \mathrm{MeV}$ ) was assumed to be unbound. Figure 3 shows the experimental $\left({ }^{19} \mathrm{C},{ }^{18} \mathrm{C}\right)$ inclusive parallel momentum distribution and also those calculated. In all cases, the theoretical momentum distribution curves are normalized to the measured inclusive cross section. We show the results obtained by (a) assuming that the $2_{3}^{+}$and $3_{1}^{+}$states are unbound (dashed curve), having an inclusive cross section of $100.2 \mathrm{mb}$, and (b) assuming that the $2_{3}^{+}$and $3_{1}^{+}$states are bound (solid curve), resulting in an inclusive cross section of $164.4 \mathrm{mb}$. The experimental cross section, in Table III, is 163(12) mb. The comparison with the present momentum distribution data, in particular, provides us with rather compelling evidence for the hypothesis (b), that the $2_{3}^{+}$and $3_{1}^{+}$states are bound.

\section{Results for ${ }^{20} \mathrm{C}$}

The predicted ${ }^{19} \mathrm{C}$ shell-model final states and the calculated and experimental one-neutron removal cross sections from the ${ }^{20} \mathrm{C}\left(0^{+}\right)$ground state, with separation energy $S_{1 n}\left({ }^{20} \mathrm{C}\right)=$ $2.90 \mathrm{MeV}$, are collected in Tables III and IV. There is only very incomplete experimental information on the low-lying excited-state spectrum of ${ }^{19} \mathrm{C}$. Using Coulomb dissociation, the ${ }^{19} \mathrm{C}$ ground state has been unambiguously identified as a $1 / 2^{+} s$-wave halo state with weak binding [20]. The evaluated ${ }^{19} \mathrm{C}$ first neutron threshold is at $0.58(9) \mathrm{MeV}$ [21]. An unbound excited $5 / 2^{+}$state with $E_{\mathrm{x}}=1.46(10) \mathrm{MeV}$ has also been clearly identified [49] using inverse-kinematics proton inelastic scattering from ${ }^{19} \mathrm{C}$. Stanoiu et al. [48] reported a 201(15) keV gamma-ray transition in ${ }^{19} \mathrm{C}$ with in-beam $\gamma$-ray spectroscopy. Using inverse-kinematics proton inelastic scattering Elekes et al. [50] also reported evidence of two gamma-ray transitions, with energies 72(4) and 197(6) $\mathrm{keV}$, from two bound ${ }^{19} \mathrm{C}$ excited states. While in both of these cases the transition energy (near $200 \mathrm{keV}$ ) is close to that of a predicted $5 / 2^{+}$shell-model bound excited state, we will show that the present experimental data and analysis do not support such a strong transition to a $5 / 2^{+}$bound ${ }^{19} \mathrm{C}$ excited state.

Table III shows the cross section for the $1 / 2_{1}^{+}$shellmodel ground-state transition. The measured cross section, of 58(5) mb, and parallel momentum distribution to a bound ${ }^{19} \mathrm{C}$ final state (Fig. 4) are consistent with the theoretical expectations for the removal of a $2 s_{1 / 2}$ neutron (solid curve) with the tabulated shell-model spectroscopic factor of near to unity. It is likely that the $1 / 2^{+}$ground state of ${ }^{19} \mathrm{C}$ is the only bound state of this system. The first $5 / 2_{1}^{+}$state in ${ }^{19} \mathrm{C}$, with $C^{2} S=3.649$, is predicted to be strongly populated with cross section $111.17 \mathrm{mb}$, far larger than the experimentally observed 58(5) mb. If any bound excited state exists, the only possibility seems to be the first $3 / 2_{1}^{+}$state predicted at $0.624 \mathrm{MeV}$, which would add the cross section of only $7.20 \mathrm{mb}$.

Although the bound $5 / 2^{+}$state is implausible due to the observed small cross section, we attempt to estimate an upper limit on possible bound $d$-state strength below. Table IV shows the results for the cross sections leading to the excited ${ }^{19} \mathrm{C}$ shell-model final states. The shell model predicts seven such excited states with significant spectroscopic factors below the ${ }^{18} \mathrm{C}$ neutron threshold of $4.18 \mathrm{MeV}$. Given these cross sections, we note, from Table IV, that one unit of the first excited state $1 d_{5 / 2}$ spectroscopic strength makes a contribution of $30.5 \mathrm{mb}$ 
including the center-of-mass correction factor $[A /(A-1)]^{N}$ to the theoretical cross section. The calculated $1 / 2_{1}^{+}$ ground-state cross section is $58.92 \mathrm{mb}$. Thus, if there was also $1 d_{5 / 2}$ strength to bound state(s), with a summed spectroscopic strength of $0.5,1.0$, or 1.5 units, the theoretical cross section to bound final states would increase to $74.2,89.4$, or $104.7 \mathrm{mb}$, respectively, well in excess of the measured value of 58(5) $\mathrm{mb}$. The corresponding effects of such bound $1 d_{5 / 2}$ strength on the shapes of the calculated ${ }^{19} \mathrm{C}$ parallel momentum distributions are shown in Fig. 4 by the long-dashed, short-dashed, and dotted-dashed curves. Here, each curve is normalized to the experimental cross section of 58(5) $\mathrm{mb}$. We conclude from this comparison that the majority of the strength that the shell-model attributes to the $190-\mathrm{keV} 1 d_{5 / 2}$ state is in fact unbound. Based on Fig. 4 and the measured cross section to bound ${ }^{19} \mathrm{C}$, we estimate that 0.5 units or less of bound $1 d_{5 / 2}$ strength might be accommodated by the present data set.

Our assumption, in Table IV, is that all of the excited ${ }^{19} \mathrm{C}$ shell-model states are unbound and that these unbound states will decay by neutron emission to ${ }^{18} \mathrm{C}$. In this and the following case of ${ }^{22} \mathrm{C}$, these unbound mass $A-1$ excitedstate cross sections are large. For such unbound final-state cases, our one-neutron removal model calculates the exclusive parallel momentum distributions of the (weakly) unbound ${ }^{19} \mathrm{C}$ and ${ }^{21} \mathrm{C}$ residues in the original projectile rest frame. The subsequent in-flight neutron emission from these excited states will generate additional (recoil) broadening of the momentum distributions of the observed mass $A-2$ residues, i.e., ${ }^{18} \mathrm{C}$ and ${ }^{20} \mathrm{C}$. The degree of broadening will be dependent on the continuum energy of the unbound mass $A-1$ intermediate state, denoted $\varepsilon^{*}$.

We estimate the effect of this recoil. We assume that, in the rest frame of the unbound mass $A-1$ state, with its given continuum energy $\varepsilon^{*}$, the mass $A-2$ residue (in its ground state) and the decay neutron separate isotropically. The momentum $p$ of the heavy decay residue in this frame satisfies $p^{2}=2 \mu \varepsilon^{*}$, where $\mu$ is the $A-2$ residue-neutron reduced mass. The assumption that this two-body decay is isotropic then requires that the calculated parallel momentum distributions of the unbound mass $A-1$ fragments must be convoluted with a rectangular distribution, of unit integral and total width $2 p$, to derive the mass $A-2$ fragment parallel momentum distributions. This is done for the theoretical distributions shown in Figs. 5 and 6.

Table IV shows that these indirect two-neutron removal cross sections arise predominantly from intermediate states of small $\varepsilon^{*}$. For the ${ }^{20} \mathrm{C}$ case, a cross section of $160 \mathrm{mb}$ is predicted to arise from the first three shell-model excited states having $E_{\mathrm{x}}<1 \mathrm{MeV}$ in ${ }^{19} \mathrm{C}$. However, as was discussed above, these shell-model energies are not sufficiently accurate. The WBP interaction predicts the first excited $5 / 2_{1}^{+}$state to be bound with a large spectroscopic factor, whereas the calculated $\sigma_{-1 n}^{\text {th }}$ to this state and the measured cross section and momentum distribution to the ${ }^{19} \mathrm{C}$ ground state exclude this possibility. Experimentally, a ${ }^{19} \mathrm{C}$ excited $5 / 2^{+}$state has been clearly identified at $E_{\mathrm{x}}=1.46(10) \mathrm{MeV}$ by Satou et al. [49] in proton inelastic scattering from ${ }^{19} \mathrm{C}$, i.e., with $\varepsilon^{*}=0.88 \mathrm{MeV}$. The present inclusive data do not permit a more detailed analysis of this excited state or of the predicted shell-model strength dis- tributions. Our analysis shows, however, that the present data are consistent with an integrated $5 / 2^{+}$strength of about 4 units leading to the ${ }^{19} \mathrm{C}$ continuum, as is given by the shell model.

In the absence of more complete information, and to assess this recoil sensitivity, we calculate the evaporation recoil effects assuming that the most important contributions arise from ${ }^{19} \mathrm{C}$ intermediate states with (i) $\varepsilon^{*}=1.0 \mathrm{MeV}$ and (ii) $\varepsilon^{*}=2.0 \mathrm{MeV}$. The results are shown in Fig. 5 by the dashed line and solid line, respectively. Both outcomes are consistent with the experimental ${ }^{18} \mathrm{C}$ residue momentum distribution. We conclude from this agreement of the inclusive cross section and momentum distribution that the $5 / 2_{1}^{+}$shell-model excited state is very likely to be unbound. Since, in this case, the shell model appears to systematically produce states with too small an excitation energy, the effective neutron separation energies will also be underestimated and, in turn, the theoretical removal cross sections slightly overestimated. We do not attempt to make any parameter adjustments to compensate for this (small) effect.

\section{Results for ${ }^{22} \mathrm{C}$}

Here, all final states of the ${ }^{21} \mathrm{C}$ one-neutron removal residues are particle unbound. The calculated exclusive (and inclusive) and experimental inclusive one-neutron removal yields are collected in Table IV for the predicted shell-model states of ${ }^{21} \mathrm{C}$, which decay by neutron emission to ${ }^{20} \mathrm{C}$.

Very little is known about these isotopes. Both the oneand two-neutron separation energies from ${ }^{22} \mathrm{C}$ are only poorly determined and so we are guided by the 2003 mass evaluation [21]. That is, $S_{2 n}\left({ }^{22} \mathrm{C}\right)=0.42(94) \mathrm{MeV}$ and $S_{1 n}\left({ }^{21} \mathrm{C}\right)=$ $-0.33(56) \mathrm{MeV}$, with large uncertainties. Thus, the ground state of ${ }^{21} \mathrm{C}$ was assumed to be produced at a continuum energy of $\varepsilon^{*}=0.30 \mathrm{MeV}$ after neutron removal with ground-state separation energy $S_{1 n}\left({ }^{22} \mathrm{C}\right)=0.70 \mathrm{MeV}$. As was discussed for the ${ }^{20} \mathrm{C}$ projectile case, the inclusive (unbound) ${ }^{21} \mathrm{C}$ momentum distribution is calculated as the weighted sum of the momentum distributions to the individual final states with the $\sigma_{-1 n(e)}^{\text {th }}$ shown in Table IV. The neutron emission recoil broadening is included for each final state according to its $\varepsilon^{*}$ value, i.e., $\varepsilon^{*}=E_{\mathrm{x}}+0.30 \mathrm{MeV}$, prior to this sum being performed. Three final states are predicted below the ${ }^{20} \mathrm{C}$ first neutron threshold of $2.90 \mathrm{MeV}$.

Table IV shows that, based on the shell model, the first two final states each contribute almost half of the inclusive one-neutron removal cross section. These states are a $1 / 2_{1}^{+}$ ground state, with spectroscopic factor $C^{2} S=1.4$, and a $5 / 2_{1}^{+}$ neutron-hole state at $E_{\mathrm{x}}=1.11 \mathrm{MeV}$, with $C^{2} S=4.2$. The associated measured and theoretical inclusive ${ }^{20} \mathrm{C}$ parallel momentum distributions (convoluted with the experimental resolution of $27 \mathrm{MeV} / c$ ) are compared in Fig. 6. The individual contributions from the two dominant shell-model final states are also shown in the figure. The agreement with the data is very good, providing strong support for the weakly bound $\nu s_{1 / 2}^{2}$ character for the ${ }^{22} \mathrm{C}$ ground state. This result is consistent with the recent interaction-cross-section measurement and associated analysis of Ref. [22], which is suggestive of an extended ${ }^{22} \mathrm{C}$ matter density. 


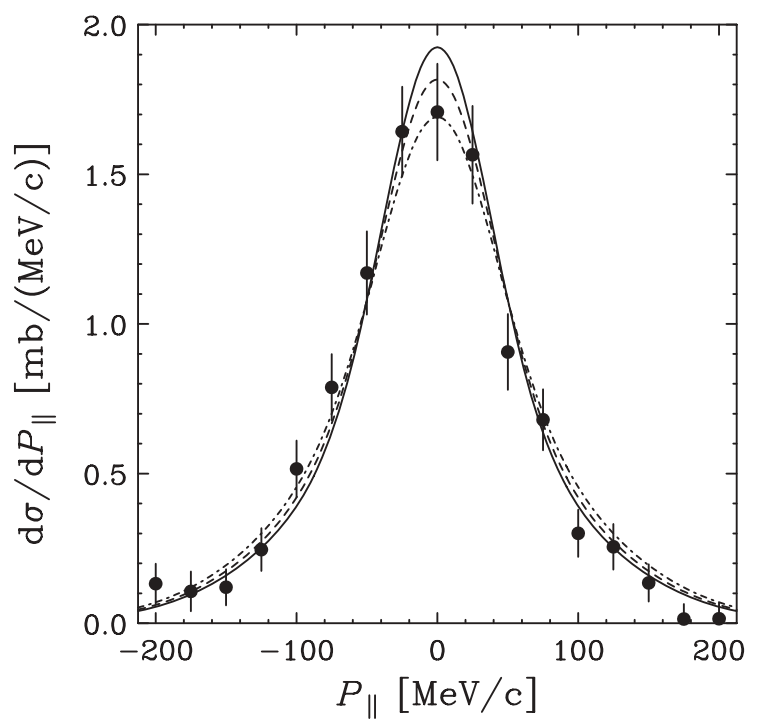

FIG. 7. Measured inclusive parallel momentum distribution of ${ }^{20} \mathrm{C}$, following two-neutron removal from ${ }^{22} \mathrm{C}$ on a carbon target at $240 \mathrm{MeV} /$ nucleon compared to the theoretical calculations. The theoretical curves are the inclusive cross sections calculated assuming ${ }^{22} \mathrm{C}$ two-neutron separation energies $S_{2 n}\left({ }^{22} \mathrm{C}\right)=0.40 \mathrm{MeV}$ (solid curve), $0.70 \mathrm{MeV}$ (dashed curve), and $1.20 \mathrm{MeV}$ (dotted-dashed curve). The curves include the recoil broadening arising from the neutron decay of the unbound ${ }^{21} \mathrm{C}$ intermediate states.

Currently, the ${ }^{22} \mathrm{C}$ two-neutron separation energy $S_{2 n}\left({ }^{22} \mathrm{C}\right)=0.42(94) \mathrm{MeV}$ has a significant uncertainty. Hence, we consider the sensitivity of the theoretical inclusive ${ }^{20} \mathrm{C}$ production cross section and momentum distribution to the value assumed. Figure 7 shows the calculated momentum distributions when assuming a ${ }^{22} \mathrm{C}$ two-neutron separation energy of $S_{2 n}\left({ }^{22} \mathrm{C}\right)=0.40 \mathrm{MeV}$ (solid curve), $0.70 \mathrm{MeV}$ (dashed curve), and $1.20 \mathrm{MeV}$ (dotted-dashed curve). In these calculations, we continue to assume that the ground state of ${ }^{21} \mathrm{C}$ is at a continuum energy of $0.30 \mathrm{MeV}$, hence, the ground-state-to-ground-state one-neutron separation energy of ${ }^{22} \mathrm{C}$ is $S_{1 n}\left({ }^{22} \mathrm{C}\right)=0.70,1.00$, and $1.50 \mathrm{MeV}$ in these cases. The curves have been convoluted with the experimental resolution of $27 \mathrm{MeV} / c$ and also include the recoil broadening arising from the neutron decay of the unbound ${ }^{21} \mathrm{C}$ states (Table IV). The increasing separation energies reduce the corresponding inclusive cross sections: 283,257 , and $227 \mathrm{mb}$ for the $S_{2 n}\left({ }^{22} \mathrm{C}\right)=0.40,0.70$, and $1.20 \mathrm{MeV}$, respectively. We note that, owing to the relative insensitivity of our calculated cross sections and momentum distributions to the current experimental uncertainty in $S_{2 n}\left({ }^{22} \mathrm{C}\right)$, the data of this work do not determine or place a significant constraint upon this value. We are able to conclude, however, that the sensitivity to the underlying structure of ${ }^{22} \mathrm{C}$, specifically of the approximately equal contributions of the $1 / 2_{1}^{+}$and $5 / 2_{1}^{+}$transitions to the measured inclusive cross section, is robust.

\section{B. Direct two-neutron removal}

We summarize only briefly the calculated exclusive and inclusive direct two-neutron removal cross sections $\sigma_{-2 n(d)}^{\text {th }}$ from
TABLE V. Theoretical results for the direct two-neutron removal reaction cross sections $\sigma_{-2 n(d)}^{\text {th }}$. Tabulated are the two-neutron removal cross sections to all predicted shell-model states below the neutron threshold in the mass $A-2$ systems ${ }^{18,20} \mathrm{C}$ (4.18 and $2.90 \mathrm{MeV}$, respectively).

\begin{tabular}{lccc}
\hline \hline Reaction & $E_{\mathrm{x}}(\mathrm{MeV})$ & $J^{\pi}$ & $\sigma_{-2 n(d)}^{\text {th }}(\mathrm{mb})$ \\
\hline$\left.{ }^{20} \mathrm{C}\left(0^{+}\right),{ }^{18} \mathrm{C}\left(J^{\pi}\right)\right]$ & 0.000 & $0_{1}^{+}$ & 5.66 \\
$S_{2 n}\left({ }^{20} \mathrm{C}\right)=3.51 \mathrm{MeV}$ & 2.114 & $2_{1}^{+}$ & 4.00 \\
& 3.639 & $2_{2}^{+}$ & 0.53 \\
& 3.988 & $0_{2}^{+}$ & 0.36 \\
& $4.915^{\mathrm{a}}$ & $3_{1}^{+}$ & 1.98 \\
& $4.975^{\mathrm{a}}$ & $2_{3}^{+}$ & 2.10 \\
& & Inclusive & 14.6 \\
{$\left[{ }^{22} \mathrm{C}\left(0^{+}\right),{ }^{20} \mathrm{C}\left(J^{\pi}\right)\right]$} & 0.000 & $0_{1}^{+}$ & 5.32 \\
$S_{2 n}\left({ }^{22} \mathrm{C}\right)=0.40 \mathrm{MeV}$ & 2.102 & $2_{1}^{+}$ & 6.81 \\
& & Inclusive & 12.1 \\
\hline \hline
\end{tabular}

${ }^{\text {aThe }}{ }^{18} \mathrm{C} 2_{3}^{+}$and $3_{1}^{+}$states at 4.915 and $4.975 \mathrm{MeV}$ are assumed to be bound (see Sec. V A1).

${ }^{20} \mathrm{C}$ and ${ }^{22} \mathrm{C}$ to bound states of the mass $A-2$ isotopes. These results are collected in Table V, computed based on the WBP shell-model two-nucleon amplitudes (TNA). As noted earlier, as these calculated cross sections were both expected and found to be small, we will not enter into an extended discussion and details of the calculations. The descriptions of the nucleon overlap functions used and the construction of the residue- and neutron-target $S$ matrices are the same as for the one-neutron removal analysis. For the full details and the formalism of the exclusive cross sections (and their momentum distributions), the reader is referred to recent references $[12,16]$.

For ${ }^{20} \mathrm{C}$ projectiles, six states below the neutron threshold in ${ }^{18} \mathrm{C}$ have appreciable TNA. These states include the $2_{3}^{+}$and $3_{1}^{+}$ states proposed as being bound from the one-neutron removal analysis (Sec. V A1). The inclusive direct two-neutron removal cross section is calculated to be $14.6 \mathrm{mb}$. For ${ }^{22} \mathrm{C}$ projectiles, just two states below the neutron threshold in ${ }^{20} \mathrm{C}$ have appreciable TNA and the direct two-neutron removal cross section is now $12.1 \mathrm{mb}$. These numbers are to be compared with those for the indirect two-neutron removal paths that predict cross sections of 191.2 and $283.0 \mathrm{mb}$, respectively. In addition, we note that, in the case of the removal of strongly bound two-neutron pairs, these calculated direct two-nucleon removal cross sections typically overestimate the measured cross sections with $R_{s}(2 N)=\sigma_{-2 n}^{\exp } / \sigma_{-2 n(d)}^{\text {th }} \approx 0.5$ [12]. Thus, as was found in the earlier study of the lighter carbon isotopes [18], the direct pathways enter at about an $8 \%$ level. Since we are unable to distinguish these direct events with the current experimental setup, they can not be elucidated or exploited further here.

\section{CONCLUSIONS}

In summary, the structure of the most neutron-rich carbon isotopes ${ }^{19,20,22} \mathrm{C}$ has been investigated using high-energy (around $240 \mathrm{MeV} /$ nucleon) single- and two-neutron removal reactions on a carbon target. Narrow momentum distributions were observed for one-neutron removal from ${ }^{19} \mathrm{C}$ and ${ }^{20} \mathrm{C}$ and two-neutron removal from ${ }^{22} \mathrm{C}$. A much broader momentum 
distribution was found in the case of two-neutron removal from ${ }^{20} \mathrm{C}$.

The measured cross sections and momentum distributions were interpreted in the light of eikonal-reaction-model calculations for single-neutron knockout combined with structural input derived from $p s d$ shell-model calculations employing the WBP interaction. The two-neutron removal cross sections were calculated by considering (a) the removal of one neutron to unbound states in the $A-1$ daughter, with the assumption that these unbound intermediate states decay by neutron emission to bound states in the mass $A-2$ residue, and (b) direct two-neutron removal.

In the case of $\mathrm{C}\left({ }^{22} \mathrm{C},{ }^{20} \mathrm{C}\right)$, the cross section and momentum distribution are consistent with the existence of a two-neutron halo with a dominant $v s_{1 / 2}^{2}$ configuration in ${ }^{22} \mathrm{C}$. The narrow momentum distribution and relatively low cross section for $\mathrm{C}\left({ }^{20} \mathrm{C},{ }^{19} \mathrm{C}\right)$, which arise as the single-neutron removal to the ${ }^{19} \mathrm{C}\left(1 / 2^{+}\right)$ground state, probe specifically the significant $v s_{1 / 2}^{2}$ component of the ${ }^{20} \mathrm{C}$ ground state. The $v d_{5 / 2}$ component results in the population of unbound states in ${ }^{19} \mathrm{C}$ that neutron decay to ${ }^{18} \mathrm{C}$. The narrow momentum distribution and enhanced cross section for $\mathrm{C}\left({ }^{19} \mathrm{C},{ }^{18} \mathrm{C}\right)$ are consistent with the well-developed $v s_{1 / 2}$ halo of ${ }^{19} \mathrm{C}$.

Overall, the calculated cross sections agreed well with those measured. In particular, in the cases of $\mathrm{C}\left({ }^{19} \mathrm{C},{ }^{18} \mathrm{C}\right), \mathrm{C}\left({ }^{20} \mathrm{C}\right.$, $\left.{ }^{19} \mathrm{C}\right)$, and $\mathrm{C}\left({ }^{22} \mathrm{C},{ }^{20} \mathrm{C}\right), R_{s}=\sigma_{-1 n}^{\exp } / \sigma_{-1 n}^{\text {th }}$ was close to unity and consistent with systematics [8]. Combined with the good agreement for the momentum distributions, it may be seen that the shell model has predictive power in this region and provides a good overall description of level positions and their spectroscopic strengths.

In the case of weakly bound two-neutron removal, fully correlated, direct removal cross sections were also calculated.
It was shown that two-neutron removal is dominated by the two-step process; that is, one-neutron removal followed by neutron decay of the unbound intermediate state(s). In the cases of two-neutron removal from ${ }^{20,22} \mathrm{C}$, the direct removal contribution was computed to be of order $8 \%$ or less of the two-step process, consistent with an earlier work [18]. Although this makes the identification of these direct twonucleon removal events challenging, kinematically complete measurements of ${ }^{20,22} \mathrm{C}$ breakup are expected to be possible in the near future and will help clarify and quantify this process.

\section{ACKNOWLEDGMENTS}

We would like to thank the RIKEN accelerator staff and BigRIPS team for the excellent beam delivery. This work was supported by the Grant-in-Aid for Scientific Research (B) (No. 22340053) from the Ministry of Education, Culture, Sports, Science and Technology (MEXT) Japan, the Global Center of Excellence Program "Nanoscience and Quantum Physics," the United Kingdom Science and Technology Facilities Council (STFC) under Grants No. ST/F012012 and No. ST/J000051, and NRF Grants No. R32-2008-000-10155-0 (WCU) and No. 2010-0027136 of MEST Korea. N.K. thankfully acknowledges the Grant-in-Aid for JSPS Fellows (No. 22.9675) from MEXT. J.A.T. gratefully acknowledges the financial and facilities support of the Department of Physics, Tokyo Institute of Technology, and E.C.S. the support of the United Kingdom Engineering and Physical Sciences Research Council (EPSRC) under Grant No. EP/P503892/1. J.G. and N.A.O. acknowledge partial support from the Franco-Japanese Nuclear Structure Program LIA.
[1] T. Kobayashi, O. Yamakawa, K. Omata, K. Sugimoto, T. Shimoda, N. Takahashi, and I. Tanihata, Phys. Rev. Lett. 60, 2599 (1988).

[2] N. A. Orr et al., Phys. Rev. Lett. 69, 2050 (1992).

[3] Y. Ogawa, Y. Suzuki, and K. Yabana, Nucl. Phys. A 571, 784 (1994).

[4] P. G. Hansen, Phys. Rev. Lett. 77, 1016 (1996).

[5] F. Barranco and E. Vigezzi, in Break-up of Halo States Induced by Nuclear Interactions, edited by R. A. Broglia and P. G. Hansen (World Scientific, Singapore, 1998), p. 217.

[6] P. G. Hansen and J. A. Tostevin, Annu. Rev. Nucl. Part. Sci. 53, 219 (2003), and references therein.

[7] C. A. Bertulani and P. G. Hansen, Phys. Rev. C 70, 034609 (2004).

[8] A. Gade et al., Phys. Rev. C 77, 044306 (2008).

[9] D. Bazin et al., Phys. Rev. Lett. 91, 012501 (2003).

[10] J. A. Tostevin, G. Podolyák, B. A. Brown, and P. G. Hansen, Phys. Rev. C 70, 064602 (2004).

[11] K. Yoneda et al., Phys. Rev. C 74, 021303 (2006).

[12] J. A. Tostevin and B. A. Brown, Phys. Rev. C 74, 064604 (2006).

[13] A. Gade et al., Phys. Rev. Lett. 99, 072502 (2007).

[14] P. Fallon et al., Phys. Rev. C 81, 041302 (2010).

[15] J. A. Tostevin, Eur. Phys. J. Spec. Top. 150, 67 (2007).
[16] E. C. Simpson, J. A. Tostevin, D. Bazin, B. A. Brown, and A. Gade, Phys. Rev. Lett. 102, 132502 (2009); E. C. Simpson, J. A. Tostevin, D. Bazin, and A. Gade, Phys. Rev. C 79, 064621 (2009).

[17] E. C. Simpson and J. A. Tostevin, Phys. Rev. C 82, 044616 (2010).

[18] E. C. Simpson and J. A. Tostevin, Phys. Rev. C 79, 024616 (2009).

[19] E. Sauvan et al., Phys. Lett. B 491, 1 (2000), and references therein.

[20] T. Nakamura et al., Phys. Rev. Lett. 83, 1112 (1999), and references therein.

[21] G. Audi, A. H. Wapstra, and C. Thibault, Nucl. Phys. A 729, 337 (2003).

[22] K. Tanaka et al., Phys. Rev. Lett. 104, 062701 (2010).

[23] D. Bazin et al., Phys. Rev. C 57, 2156 (1998).

[24] T. Baumann et al., Phys. Lett. B 439, 256 (1998).

[25] A. Ozawa et al., Phys. Rev. C 78, 054313 (2008).

[26] V. Maddalena et al., Phys. Rev. C 63, 024613 (2001).

[27] T. Yamaguchi et al., Nucl. Phys. A 724, 3 (2003).

[28] E. Sauvan et al., Phys. Rev. C 69, 044603 (2004).

[29] J. R. Terry et al., Phys. Rev. C 69, 054306 (2004).

[30] M. Chiba et al., Nucl. Phys. A 741, 29 (2004). 
[31] C. Wu et al., J. Phys. G: Nucl. Part. Phys. 31, 39 (2005).

[32] Y. Kondo et al., Phys. Rev. C 79, 014602 (2009).

[33] A. Ozawa et al., Phys. Rev. C 84, 064315 (2011).

[34] T. Kubo, Nucl. Instrum. Methods Phys. Res., Sect. B 204, 97 (2003).

[35] T. Ohnishi et al., J. Phys. Soc. Jpn. 77, 083201 (2008).

[36] K. Hencken, G. Bertsch, and H. Esbensen, Phys. Rev. C 54, 3043 (1996).

[37] A. S. Goldhaber, Phys. Lett. B 53, 306 (1974).

[38] H. Esbensen and G. F. Bertsch, Phys. Rev. C 64, 014608 (2001); G. F. Bertsch and H. Esbensen, Prog. Theor. Phys. (Suppl.) 146, 319 (2002).

[39] J. A. Tostevin, Nucl. Phys. A 682, 320c (2001).
[40] J. S. Al-Khalili, J. A. Tostevin, and I. J. Thompson, Phys. Rev. C 54, 1843 (1996).

[41] B. A. Brown, Phys. Rev. C 58, 220 (1998).

[42] L. Ray, Phys. Rev. C 20, 1857 (1979).

[43] D. Bazin et al., Phys. Rev. Lett. 102, 232501 (2009).

[44] C. A. Bertulani and C. De Conti, Phys. Rev. C 81, 064603 (2010).

[45] B. A. Brown et al., Oxbash for Windows (2004), MSU-NSCL Report No. 1289 (unpublished).

[46] E. K. Warburton and B. A. Brown, Phys. Rev. C 46, 923 (1992).

[47] A. E. L. Dieperink and T. de Forest, Jr., Phys. Rev. C 10, 543 (1974).

[48] M. Stanoiu et al., Phys. Rev. C 78, 034315 (2008).

[49] Y. Satou et al., Phys. Lett. B 660, 320 (2008).

[50] Z. Elekes et al., Phys. Lett. B 614, 174 (2005). 